TRANSACTIONS OF THE

AMERICAN MATHEMATICAL SOCIETY

\title{
PURE WEAK MIXING
}

BY

R. ELLIS ${ }^{1}$ AND S. GLASNER

\begin{abstract}
We show that for the group of integers $\mathbf{Z}$, the maximal common factor of the maximal weakly mixing minimal flows coincides with the universal purely weakly mixing flow. We show that the maximal weakly mixing minimal flows are not all isomorphic to each other. None of the maximal w.m. flows are regular.
\end{abstract}

0. Introduction. In his paper Some extensions of weakly mixing flows, $\mathbf{R}$. Peleg has shown, using the method developed in [3], that for almost every cocycle $\sigma$ defined on a metric minimal weakly mixing (w.m.) flow $(Y, \tau)(Y$ is a compact metric space and $\tau$ is a homeomorphism of $Y$ which defines the flow) with values in a compact group $K$, the resulting flow on $Y \times K$ is minimal and w.m. He then used this construction to deduce the existence of two w.m. minimal flows $(X, \tau)$ and $(Y, \tau)$ such that some minimal subset of the product flow $(X \times Y, \tau)$ is not w.m. He observed, however, that if for any two w.m. minimal flows $(X, \tau)$ and $(Y, \tau)$, some minimal set in the product $(X \times Y, \tau)$ were to be w.m. then there would necessarily exist a universal w.m. minimal flow i.e. a w.m. minimal flow which has all w.m. minimal flows as its factors.

We use the same letter $\tau$ to denote the generating homeomorphism of any flow and thus omit it in the notation and speak simply of the flows $X, Y$ etc.

In order to gain a better understanding of the problems we are about to pose, it is necessary to introduce the notion of a pointed flow. This is a flow $X$ together with a distinguished point $x_{0} \in X$. Denote this by $\left(X, x_{0}\right)$. We define the pointed product of two pointed flows $\left(X, x_{0}\right)$ and $\left(Y, y_{0}\right)$ to be the pointed flow whose phase space is the orbit closure of $\left(x_{0}, y_{0}\right)$ in $X \times Y$ and whose distinguished point is $\left(x_{0}, y_{0}\right)$. We write $\left(X, x_{0}\right) \vee\left(Y, y_{0}\right)$ for this product.

In order that the pointed product of two minimal flows will be again a minimal flow, we restrict ourselves to pointed flows $\left(X, x_{0}\right)$ where $x_{0}$ is such that $x_{0} u=x_{0}$. Here $u$ is a fixed idempotent in a fixed minimal ideal of the Stone-Čech compactification of $\left\{\tau^{n}: n \in \mathbf{Z}\right\}$. This does not restrict the generality. Our category then is that of pointed minimal flows whose

Received by the editors January 25, 1977.

AMS (MOS) subject classifications (1970). Primary 54H20.

Key words and phrases. Minimal flow, weak mixing, regular, extension, group of a flow.

${ }^{1}$ Partially supported by NSF Grant MPS 75-05250.

- American Mathematical Society 1978 
distinguished point is fixed by $u$. The morphisms of this category are homomorphisms of flows which send distinguished points into distinguished points. Notice that at most one such homomorphism exists between a pair of pointed minimal flows. In this category we have products and also intersections of any number of pointed minimal flows. Thus if $\left\{\left(X_{\mu}, x_{\mu}\right)\right\}$ is a family of pointed flows then $\bigvee\left(X_{\mu}, x_{\mu}\right)$ is the orbit closure of the point $\left(x_{\mu}\right) \in \Pi X_{\mu}$ with distinguished point $\left(x_{\mu}\right) \cdot \wedge\left(X_{\mu}, x_{\mu}\right)$ is the unique maximal common factor of all the flows $\left(X_{\mu}, x_{\mu}\right)$.

Define an equivalence relation on w.m. pointed minimal flows: $\left(X, x_{0}\right) \sim$ $\left(Y, y_{0}\right)$ iff $\left(X, x_{0}\right) \vee\left(Y, y_{0}\right)$ is w.m. Let $I$ be the set of equivalence classes. It follows from Peleg's result that $I$ is infinite. For each equivalence class $i \in I$ let $\left(X_{i}, x_{i}\right)=\bigvee_{\mu \in i}\left(X_{\mu}, x_{\mu}\right)$. We call $\left(X_{i}, x_{i}\right)$ a maximal w.m. pointed minimal flow. It is easy to see that a pointed minimal flow $\left(Y, y_{0}\right)$ is a factor of $\bigwedge_{i \in I}\left(X_{i}, x_{i}\right)$ iff for every pointed w.m. minimal flow $\left(Z, z_{0}\right)$, the minimal flow $\left(Y, y_{0}\right) \vee\left(Z, z_{0}\right)$, is w.m. Thus $\bigwedge\left(X_{i}, x_{i}\right)$ is the universal pointed minimal flow for this property. We are now ready to pose our first problem.

(1) Identify $\bigwedge_{i \in I}\left(X_{i}, x_{i}\right)$. In particular is this the trivial one point flow? Let $\left(X_{i}, x_{i}\right)$ be one of the maximal w.m. pointed minimal flows and let $x \in X$ with $x u=x$. Then $\left(X_{i}, x\right)$ is a w.m. pointed minimal flow and for a unique $j \in I$ there exists a homomorphism $\left(X_{j}, x_{j}\right) \stackrel{\varphi}{\rightarrow}\left(X_{i}, x\right)$. Since for some $y \in X_{j}$, $\varphi(y)=x_{i}$ we have $\left(X_{j}, y\right) \stackrel{\varphi}{\rightarrow}\left(X_{i}, x_{i}\right)$. This implies that $\left(X_{j}, y\right) \in i$ and by the maximality of $\left(X_{i}, x_{i}\right), \varphi$ is an isomorphism. Thus some of the flows $X_{i}$ may be isomorphic to each other, though of course not as pointed flows. This divides $I$ into subclasses as follows: $i$ and $j$ are in the same subclass if there exists an isomorphism of $X_{i}$ onto $X_{j}$. Our next problem is the following.

(2) How many subclasses are there in I? In particular is there only one subclass; i.e. are all the $\left\{X_{i}\right\}_{i \in I}$ isomorphic? We notice that this is Peleg's question about the existence of a universal w.m. minimal flow.

A minimal flow $X$ is called regular if for every pair of points $x, y \in X$ with $y u=x, y u=y$ there exists an isomorphism $(X, x) \rightarrow(X, y)$. The statement " $X_{i}$ is regular" is equivalent therefore to the fact that the subclass of $i$ in $I$ is the singleton $\{i\}$.

(3) Are any of the flows $X_{i}$ regular? Are they all regular? One can easily see that the answer to the latter question is affirmative iff for every w. m. minimal flow $X$ every minimal subset of $X \times X$ is w.m.

In this paper we solve the problems 1-3 as follows. The answer to problem 3 is negative. None of the flows $X_{i}$ is regular. We also show that there exists a metric w.m. minimal flow $X$ such that in $X \times X$ there exists a minimal subflow which is not w.m. The answer to problem 2 is that there are many different subclasses of $I$. In particular there does not exist a universal w.m. minimal flow and there are w.m. minimal flows $X$ and $Y$ such that every 
minimal subset of $X \times Y$ is not w.m. This answers Peleg's question in [8].

We now turn to the first problem. We say that a pointed minimal flow $\left(X, x_{0}\right)$ is purely weak mixing (p.w.m.) if for every pointed minimal flow $\left(Y, y_{0}\right)$ and homomorphisms $\left(X, x_{0}\right) \vee\left(Y, y_{0}\right) \stackrel{\varphi}{\rightarrow}\left(Z, z_{0}\right) \stackrel{\psi}{\rightarrow}\left(Y, y_{0}\right)$ where $\psi$ is an almost periodic homomorphism, $\psi$ must be an isomorphism. Taking $\left(Y, y_{0}\right)$ to be the trivial one point flow, we see that a p.w.m. flow is w.m. Moreover if $\left(X, x_{0}\right)$ is p.w.m. then there does not exist a diagram $\left(X, x_{0}\right) \rightarrow$ $\left(Z, z_{0}\right) \rightarrow\left(Y, y_{0}\right)$ with $\left(Z, z_{0}\right) \rightarrow\left(Y, y_{0}\right)$ a nontrivial almost periodic extension. Our main result is that there exists a universal p.w.m. minimal flow which is regular, and that this universal flow coincides with $\bigwedge_{i \in I}\left(X_{i}, x_{i}\right)$. Moreover we show that every P.O.D. flow (see definition in the next section) is p.w.m.; thereby showing that $\bigwedge_{i \in I}\left(X_{i}, x_{i}\right)$ is nontrivial. A notion of p.w.m. appeared first in [9].

1. The algebraic setting. In the rest of this paper we shall use the algebraic methods and language of topological dynamics as developed in [2], [5] and [4]. We shall describe briefly the correspondence between our former description and the algebraic one and will then restate problems 1-3 in algebraic terms. For a more detailed exposition we refer the reader to [2] and [6].

Given a pointed flow $\left(X, x_{0}\right)$ we associate with each continuous function $f$ on $X$ a bounded function $\tilde{f}$ on $\mathbf{Z}$, the group of integers, as follows $\tilde{f}(n)=$ $f\left(x_{0} \tau^{n}\right)$. In this way we associate with every pointed flow $\left(X, x_{0}\right)$ a shift invariant, closed, algebra $\mathcal{Q}$ of bounded functions on $\mathbf{Z}$. If the orbit of $x_{0}$ is dense in $X$ then the pointed flow $\left(X, x_{0}\right)$ can be retrieved from $\mathscr{Q}$ as the maximal ideal space of $\mathcal{Q}$, where the distinguished point is the functional $\tilde{f} \rightarrow \tilde{f}(0)$. We denote this pointed flow, which is isomorphic to $\left(X, x_{0}\right)$, by $|\mathbb{Q}|$. Conversely, every shift invariant, closed algebra $\mathbb{Q}$ defines a pointed flow $|\mathbb{Q}|$ whose distinguished point has a dense orbit. If $\mathbb{Q}$ corresponds to $\left(X, x_{0}\right)$ and $\mathscr{B}$ to $\left(Y, y_{0}\right)$ then $\mathscr{B} \subset \mathbb{Q}$ iff $\left(X, x_{0}\right) \rightarrow\left(Y, y_{0}\right)$. Let $\left\{\mathbb{Q}_{\mu}\right\}$ be a family of shift invariant closed algebras, let $\bigvee \mathbb{Q}_{\mu}$ be the shift invariant closed algebra generated by all the $\mathbb{Q}_{\mu}$ and let $\cap \mathbb{Q}_{\mu}$ be their intersection; then

$$
\left|\bigvee \mathbb{Q}_{\mu}\right| \cong \bigvee\left|\mathbb{Q}_{\mu}\right| \text { and }\left|\cap \mathbb{Q}_{\mu}\right| \cong \wedge\left|\mathbb{Q}_{\mu}\right|
$$

as pointed flows.

Let $\beta \mathbf{Z}$ be the Stone-Čech compactification of $\mathbf{Z}$. As usual we let $M$ be a minimal right ideal of the semigroup $\beta \mathbf{Z}, J$ its set of idempotents, $u \in J$ a distinguished idempotent and $G=M u$. On $G$ we have a compact $T_{1}$ topology, the $\tau$-topology, which is weaker than the topology induced on $G$ from $\beta \mathbf{Z}$.

$\beta \mathbf{Z}$ is a universal point transitive flow, and $M$ is a universal minimal flow. There is an action of $\beta \mathbf{Z}$ on every flow and in particular an action of $M$ on 
every minimal flow. Let $\mathfrak{A}(u)$ be the shift invariant closed algebra corresponding to the pointed flow $(M, u)$, then a shift invariant closed algebra $\mathbb{Q}$ is a subalgebra of $\mathfrak{A}(u)$ iff $\mathscr{Q}$ corresponds to a pointed minimal flow $\left(X, x_{0}\right)$ with $x_{0} u=x_{0}$. From now on we refer to shift invariant closed subalgebras of $\mathfrak{Y}(u)$ simply as algebras. Let $\left(X, x_{0}\right)$ be a pointed minimal flow with $x_{0} u=x_{0}$ then $\{x \in X: x u=x\}=\{x \alpha: \alpha \in G\}$. If $\mathcal{Q}$ corresponds to $\left(X, x_{0}\right)$ we write $\mathscr{S}(\mathbb{Q})=\mathscr{G S}\left(X, x_{0}\right)=\left\{\alpha \in G: x_{0} \alpha=x_{0}\right\}$. This is a $\tau$-closed subgroup of $G$.

Let $\tilde{f} \in \mathbb{Q}$ and $\alpha \in G$. We define $(\tilde{f} \alpha)(n)=f\left(x_{0} \alpha \tau^{n}\right)(n \in \mathbf{Z})$. We have for $x=x_{0} \alpha \in X$, $\mathbb{S}(\mathbb{Q} \alpha)=\mathbb{S}(X, x)=\alpha^{-1} \mathbb{S}(\mathbb{Q}) \alpha$. Also $\mathbb{S}\left(\bigvee \mathbb{Q}_{\mu}\right)=$ $\cap \mathbb{S}\left(\mathbb{Q}_{\mu}\right)$.

Let $B$ be the Bohr compactification of $\mathbf{Z}$. Then $B$ is the universal equicontinuous minimal flow and the algebra $\mathcal{E}$, which corresponds to $(B, e)(e$ denotes the identity element of a group), is the algebra of almost periodic functions on $\mathbf{Z}$.

Put $E=\mathscr{S}(\mathcal{E})=\mathbb{S}(B, e)$. Since our acting group is $\mathbf{Z}$, an algebra $\mathcal{Q}$ is w.m. (i.e. the corresponding flow is w.m.) iff $\mathscr{S}(\mathscr{Q}) \cdot E=G$. Let $\left\{A_{i}\right\}_{i \in I}$ be the family of $\tau$-closed subgroups of $G$ such that $A_{i} E=G$ and $A_{i}$ is minimal with respect to this property. Let $\mathfrak{A}\left(A_{i}\right)=\left\{f \in \mathfrak{A}(u): f \alpha=f, \forall \alpha \in A_{i}\right\}$, then an algebra $\mathbb{Q}$ is w.m. iff for some $i, \mathbb{Q} \subseteq \mathfrak{U}\left(A_{i}\right)$. The pointed minimal flows which correspond to the $\mathfrak{Y}\left(A_{i}\right)$ 's are the maximal pointed w.m. minimal flows $\left(X_{i}, x_{i}\right)$.

Let $\alpha \in G$ and $j \in I$, then clearly $\alpha^{-1} A_{j} \alpha \in\left\{A_{i}\right\}_{i \in I}$ and $\mathfrak{Y}\left(A_{j}\right) \alpha=$ $\mathfrak{U}\left(\alpha^{-1} A_{j} \alpha\right)$. The flows $\left|\mathfrak{U}\left(A_{j}\right)\right|$ and $\left|\mathfrak{U}\left(\alpha^{-1} A_{j} \alpha\right)\right|$ are then isomorphic. Conversely, if $\left|\mathfrak{Y}\left(A_{i}\right)\right|$ and $\left|\mathfrak{U}\left(A_{j}\right)\right|$ are isomorphic flows then there exists an $\alpha \in G$ such that $A_{i}=\alpha^{-1} A_{j} \alpha$. Finally $\left|\mathfrak{A}\left(A_{i}\right)\right|$ is regular iff $A_{i}$ is normal in $G$.

The problems 1-3 can now be formulated as follows

(1) Identify $\cap \mathfrak{U}\left(A_{i}\right)$. In particular is it just the algebra of constant functions?

(2) How many conjugacy classes are there in the family $\left\{A_{i}\right\}_{i \in I}$ ? Are they all conjugate?

(3) Are any of the groups $A_{i}$ normal? Are they all normal?

We conclude this section with some definitions and results.

Let $\mathbb{Q}$ be an algebra, we write $\mathbb{Q}^{\#}$ for the largest almost periodic extension of $\mathbb{Q}$ in $\mathfrak{Y}(u)$. If $A$ is a $\tau$-closed subgroup of $G$ then $H(A)$ is the smallest $\tau$-closed normal subgroup of $A$ such that $A / H(A)$ is Hausdorff. We have $\mathbb{S}\left(\mathbb{Q}^{\sharp}\right)=H(\mathbb{S}(\mathbb{Q}))$ and $H(G)=E$.

An extension $\mathscr{F} \subseteq \mathbb{Q}$ is called RIC if $\mathfrak{X}(F, \mathbb{Q})=\mathscr{Q}$, where $\mathfrak{A}(F, \mathbb{Q})$ is the quasifactor of $\mathbb{Q}$ generated by $F=\mathscr{E S}(\mathscr{F})$. We shall need the following theorem [5, Theorem 4.3] or [6, Theorem 8.2.1].

THEOREM 1.1. If $\mathscr{Q} \supseteq \mathbb{Q}$ is RIC then

$$
\mathfrak{S}\left(\mathscr{B} \cap \mathbb{Q}^{\sharp}\right)=B H(A) \quad(B=\mathscr{S}(\mathscr{B}), A=\mathbb{S}(\mathscr{Q})) .
$$


If $K$ is a compact $T_{2}$ topological group and $\mathscr{Q}$ an algebra then $Z(Q, K)$ is the set of cocycles on $\mathbf{Z} \times|\mathfrak{Q}|$ to $K$. If $\sigma \in Z(\mathbb{Q}, K)$ then $\operatorname{ext}(\mathcal{Q}, \sigma)$ is the algebra corresponding to the orbit closure of $\left(e, x_{0}\right) \in K \times_{\sigma}|\mathfrak{Q}|$, where $e$ is the identity of $K, x_{0}$ is the base point of $|\mathfrak{Q}|$ and the flow on $K \times_{\sigma}|\mathbb{Q}|$ is defined by $(k, x) t=(k \sigma(x, t), x t)(k \in K, x \in|\mathbb{Q}|, t \in \mathbf{Z})$.

Every $\sigma \in Z(\mathbb{Q}, K)$ defines a function $f_{\sigma}: \beta \mathbf{Z} \rightarrow K, f_{\sigma}(t)=\sigma\left(x_{0}, t\right)(t \in \mathbf{Z})$. Let $A=\mathbb{S}(\mathbb{Q})$; then $f_{\sigma} \mid A$ is a $\tau$-continuous homomorphism of $A$ into $K$ and $\mathbb{S}$ $(\operatorname{ext}(\mathbb{Q}, \sigma))=\operatorname{ker}\left(f_{\sigma} \mid A\right)$. $\operatorname{Hom}(\mathbf{Z}, K)$ is the set of homomorphisms of $\mathbf{Z}$ into $K$; it can be considered as a subset of $Z(\mathfrak{U}(u), K)(\rho(x, t)=\rho(t), \rho \in$ $\operatorname{Hom}(\mathbf{Z}, K), x \in M, t \in \mathbf{Z})[4]$.

Let $Q \subseteq \mathscr{B}$ be a group extension (i.e. $|\mathscr{B}| \rightarrow|\mathbb{Q}|$ is almost periodic, $B=\mathscr{S}(\mathscr{B})$ is a normal subgroup of $A=\mathscr{S}(\mathscr{Q})$ and $K=A / B$ is a compact $T_{2}$ topological group). If $\sigma \in Z(\mathbb{Q}, K)$ then $\operatorname{per}(\mathscr{B}, \sigma)$ is the algebra which corresponds to the orbit closure of the base point $x_{0} \in|\Re|$ under the flow $x \rightarrow \sigma(x, t)^{-1} x t(x \in|\mathbb{Q}|, t \in Z)$. We have $\mathbb{S}(\operatorname{per}(\mathscr{B}, \sigma))=\left\{\alpha \in A: f_{\sigma}(\alpha)=\right.$ $B \alpha\}$. Let $\mathscr{Q}$ and $\mathscr{B}$ be algebras, we say that $\mathscr{Q}$ is disjoint from $\mathscr{B}(\mathbb{Q} \perp \mathscr{B})$ if $|\mathfrak{Q}| \times|\mathfrak{B}|$ is a minimal flow. An algebra $\mathscr{Q}$ is called P.O.D. if $|\mathscr{Q}|$ is totally minimal and given $x, y \in|\mathbb{Q}|$ there exists an $n \in \mathbf{Z}$ such that $x \tau^{n}$ and $y$ are proximal [7].

\section{Pure weak mixing.}

Defintion. An algebra $\mathfrak{B} \subseteq \mathfrak{A}(u)$ is called purely weakly mixing (p.w.m.) if for every algebra $\mathbb{Q} \subseteq \mathfrak{A}(u)$

$$
(\mathscr{B} \vee \mathbb{Q}) \cap \mathbb{Q}^{\sharp}=\mathscr{Q} .
$$

A $\tau$-closed subgroup $B$ of $G$ is called p.w.m. if $\mathfrak{A}(B)$ is p.w.m.

Proposition 2.1. An algebra 9 is p.w.m. iff for every $A$, a $\tau$-closed subgroup of $G$,

$$
(A \cap B) H(A)=A \text {, }
$$

where $B=\mathscr{B}(\mathscr{B})$.

Proof. Assume first that $\mathscr{B}$ is p.w.m. and let $A$ be a $\tau$-closed subgroup, then

$$
\mathfrak{L}=\mathfrak{U}(A)^{\sharp} \cap(\mathfrak{Y}(A) \bigvee \mathfrak{B})=\mathfrak{A}(A) .
$$

But $\mathfrak{A}(A) \bigvee \mathfrak{B} \supseteq \mathfrak{U}(A)$ is a RIC extension hence by Theorem 1.1.

$$
\begin{aligned}
A & =\mathscr{S}(\mathfrak{A}(A))=\mathscr{S}(\mathfrak{L}) \\
& =\mathbb{S}(\mathfrak{A}(A) \vee \mathscr{B}) H(A)=(A \cap B) H(A) .
\end{aligned}
$$

Conversely suppose $(A \cap B) H(A)=A$ for every $\tau$-closed group $A$, and let $\mathbb{Q}$ be an algebra. Put $\mathcal{L}=\mathbb{Q}^{\#} \cap(\mathscr{Q} \vee \mathfrak{B})$, then $\mathcal{L}$ is an almost periodic extension of $\mathscr{Q}$ and we therefore have $\mathbb{S}(\mathfrak{L}) \supseteq H(A)$. Also $A \cap B=\mathbb{S}(\mathbb{Q} \vee$ 
B) $\supseteq \mathbb{S}(\mathscr{L})$ hence

$$
A \supseteq \circlearrowleft(\varrho) \supseteq H(A)(A \cap B)=A,
$$

implies $\mathbb{S}(\mathscr{L})=\mathscr{S}(\mathbb{Q})=A$. Since $\mathscr{L} \supseteq \mathbb{Q}$ is almost periodic this means $\mathscr{L}=$ Q.

Proposition 2.2. There exists a maximal p.w.m. algebra which contains every other p.w.m. algebra. This maximal algebra is of the form $\mathfrak{A}\left(B_{0}\right)$ where $B_{0}$ is a normal $\tau$-closed subgroup of $G$.

Proof. First observe that $G$ is p.w.m. Now let $B$ be any p.w.m. $\tau$-closed subgroup of $G$. Using Zorn's lemma we can find a minimal subgroup $B^{\prime} \supseteq B$ which is p.w.m. Let $B_{1}$ and $B_{2}$ be two minimal p.w.m. $\tau$-closed subgroups and let $\mathscr{B}_{i}=\mathfrak{U}\left(B_{i}\right)(i=1,2)$. If $\mathscr{Q}$ is an algebra then

$$
\left(\mathscr{Q} \vee \mathscr{B}_{1}\right)^{\sharp} \cap\left(\left(\mathscr{Q} \vee \mathscr{B}_{1}\right) \vee \mathscr{B}_{2}\right)=\mathscr{Q} \vee \mathscr{B}_{1}
$$

Since $\mathbb{Q}^{\sharp} \subseteq\left(\mathbb{Q} \vee \mathscr{B}_{1}\right)^{\sharp}$ we have

$$
\mathbb{Q}^{\sharp} \cap\left(\mathbb{Q} \vee\left(\mathscr{B}_{1} \vee \mathscr{B}_{2}\right)\right) \subseteq\left(\mathbb{Q} \vee \mathscr{B}_{1}\right) \cap \mathbb{Q}^{\sharp}=\mathscr{Q}
$$

Hence $\mathscr{B}_{1} \vee \mathscr{B}_{2}$ is p.w.m.; but $\leftrightarrow\left(\mathscr{B}_{1} \vee \mathscr{B}_{2}\right)=B_{1} \cap B_{2}$ and the minimality of $B_{i}(i=1,2)$ implies $B_{1}=B_{2}$. Thus there is only one minimal p.w.m. group which we denote by $B_{0}$. Clearly the maximal p.w.m. algebra is $\mathfrak{A}\left(B_{0}\right)$. It remains to show that $B_{0}$ is normal. Let $\alpha \in G$ and let $A$ be a $\tau$-closed subgroup of $G$, then

$$
\begin{aligned}
\left(\alpha B_{0} \alpha^{-1} \cap A\right) H(A) & =\left(\alpha B_{0} \alpha^{-1} \cap \alpha\left(\alpha^{-1} A \alpha\right) \alpha^{-1}\right) \alpha H\left(\alpha^{-1} A \alpha\right) \alpha^{-1} \\
& =\alpha\left(\left(B_{0} \cap\left(\alpha^{-1} A \alpha\right)\right) H\left(\alpha^{-1} A \alpha\right)\right) \alpha^{-1} \\
& =\alpha\left(\alpha^{-1} A \alpha\right) \alpha^{-1}=A .
\end{aligned}
$$

Thus $\alpha B_{0} \alpha^{-1}$ is p.w.m. and is therefore equal to $B_{0}$. This completes the proof.

PROPOSITION 2.3. Let $\mathcal{P}$ be an algebra which is not equicontinuous and such that for every algebra $\mathbb{Q}$ either $\mathcal{P} \subseteq \mathbb{Q}$ or $\mathcal{P}$ is disjoint from $\mathbb{Q}$; then $\mathcal{P}$ is p.w.m. Thus Furstenberg-Keynes-Shapiro's example of a prime flow implies that $\mathfrak{U}\left(B_{0}\right)$ is not trivial (or that $\left.B_{0} \neq G\right)$. (See [7].)

Proof. Let $\mathscr{Q}$ be given. If $\mathscr{P} \subseteq \mathbb{Q}$ then $\mathscr{Q} \vee \mathscr{P}=\mathscr{Q}$ and $\mathbb{Q}^{\sharp} \cap(\mathbb{Q} \vee \mathscr{P})=$ $\mathbb{Q}^{\sharp} \cap \mathbb{Q}=\mathbb{Q}$. Now assume $\mathbb{Q} \perp \mathscr{P}$. If $\mathscr{P} \subseteq \mathbb{Q}^{\sharp}$ then $\mathbb{Q} \subseteq \mathbb{Q} \vee \mathcal{P}$ is an almost periodic extension. Since $\mathscr{Q} \perp \mathcal{P},|\mathfrak{Q} \vee \mathscr{P}|=|\mathfrak{Q}| \times|\mathscr{P}|$ and hence $|\mathscr{P}|$ must be an equicontinuous flow. This contradicts our assumption and we therefore conclude that $\mathscr{P} \perp \mathbb{Q}^{\sharp}$.

Let $\mathcal{L}=\mathbb{Q}^{\sharp} \cap(\mathscr{Q} \vee \mathcal{P})$, then clearly $\mathscr{Q} \subseteq \mathcal{L}$. Let $p, q \in M$ we show that $p=q$ on $\mathbb{Q}$ implies $p=q$ on $\mathcal{E}$. This will show that also $\mathcal{E} \subseteq \mathbb{Q}$. Since $\mathscr{P} \perp \mathbb{Q}^{\sharp}$ there exist $r, s \in M$ such that $r=s$ on $\mathcal{P}$ and $r=p, s=q$ on $\mathbb{Q}^{\sharp}$. 
Since $\mathbb{Q} \subseteq \mathbb{Q}^{\#}$ we also have $r=p, s=q$ on $\mathbb{Q}$ and hence $r=s$ on $\mathscr{P} \vee \mathbb{Q}$ and a fortiori $r=s$ on $\mathcal{L}$. But $\mathcal{L} \subseteq \mathbb{Q}^{\#}$, hence on $\mathcal{L}, p=r=s=q$ and our proof is complete.

Proposition 2.4. $\mathfrak{A}\left(B_{0}\right) \subseteq \cap \mathfrak{A}\left(A_{i}\right)$.

PRoor. If $\mathfrak{X}\left(B_{0}\right) \nsubseteq \mathfrak{U}\left(A_{i}\right)$ then by the maximality of $\mathfrak{U}\left(A_{i}\right), \mathfrak{U}\left(B_{0}\right) \vee \mathfrak{U}\left(A_{i}\right)$ contains an almost periodic function $f$. Let $\mathbb{Q}_{f}$ be the Z-invariant closed algebra generated by $f$. Then

$$
\mathbb{Q}_{f} \vee \mathfrak{A}\left(A_{i}\right) \subseteq \mathfrak{A}\left(A_{i}\right)^{\sharp} \cap\left(\mathfrak{X}\left(A_{i}\right) \vee \mathfrak{U}\left(B_{0}\right)\right)=\mathfrak{A}\left(A_{i}\right)
$$

This contradicts the weak mixing of $\mathfrak{A}\left(A_{i}\right)$. Thus $\mathfrak{X}\left(B_{0}\right) \subseteq \mathfrak{X}\left(A_{i}\right)$ for every $i$.

3. $\mathfrak{X}\left(B_{0}\right)=\cap \mathfrak{X}\left(A_{i}\right)$.

LEMMA 3.1. Let $B$ be a $\tau$-closed normal subgroup of $G$. Then $\mathfrak{X}(B)$ is p.w.m. iff for every $\tau$-closed group $A \supseteq B$

$$
B H(A)=A \text {. }
$$

PROOF. Clearly the condition of the lemma is satisfied if $\mathfrak{A}(B)$ is p.w.m. Suppose that the condition is satisfied and let $A$ be an arbitrary $\tau$-closed subgroup of $G$. Since $B$ is normal $A B=F$ is a $\tau$-closed subgroup of $G$. Now $F \supseteq B$ hence our assumption implies $B H(F)=F$. By $[5,3.12]$

$$
F=B H(F)=B H(B A)=B H(A) \text {. }
$$

Hence

$$
A=A \cap F=A \cap B H(A)=(A \cap B) H(A) .
$$

Thus $(A \cap B) H(A)=A$ and $\mathscr{A}(B)$ is p.w.m. by Proposition 2.1.

Proposition 3.2. Let $\mathbb{Q} \subseteq \mathscr{B}$ be a group extension (i.e. $\mathbb{Q} \subseteq \mathscr{B}$ is almost periodic extension and $B=\mathbb{S}(\mathscr{B})$ is normal in $A=\mathbb{S}(\mathbb{C})$ ). Assume $\mathscr{B}$ is w.m. and let $\sigma$ be a homomorphism of $\mathbf{Z}$ into the compact topological group $A / B$. Then the algebra $\mathscr{F}=\operatorname{per}(\mathscr{B}, \sigma)$ is w.m.

Proof. Let $F=\mathbb{S}(\mathscr{F})$. By [4, Proposition 5.3] $F=\left\{\alpha \in A: f_{\sigma}(\alpha)=B \alpha\right\}$. Now let $\gamma \in G=B E$ then $\gamma=\beta \eta, \beta \in B, \eta \in E$. Let $f_{\sigma}(\beta)=B \rho ; \rho \in A$. Then $\rho=\beta_{1} \eta_{1} ; \beta_{1} \in B, \eta_{1} \in E$. Hence

$$
f_{\sigma}(\beta)=B \rho=B \beta_{1} \eta_{1}=B \eta_{1}=B \beta \eta_{1}
$$

and since $E \subseteq \operatorname{ker} f_{\sigma}, f_{\sigma}\left(\beta \eta_{1}\right)=f_{\sigma}(\beta)=B \beta \eta_{1}$, whence $\beta \eta_{1} \in F$. Thus $\gamma=$ $\beta \eta=\left(\beta \eta_{1}\right)\left(\eta_{1} \eta\right) \in F E$, and $\mathscr{F}$ is w.m.

Let $\mathfrak{X}\left(A_{i}\right)$ be one of the maximal w.m. algebras and let $\alpha \in G$, then $\left(\alpha A_{i} \alpha^{-1}\right) E=\left(\alpha A_{i} \alpha^{-1}\right)\left(\alpha E \alpha^{-1}\right)$ and $\mathscr{N}\left(A_{i}\right) \alpha$ is also a maximal w.m. algebra and hence $\left(\cap \mathscr{Y}\left(A_{i}\right)\right) \alpha=\cap \mathscr{Y}\left(A_{i}\right)$. Let $B_{1}=\mathbb{S}\left(\cap \mathfrak{A}\left(A_{i}\right)\right)$ then $B_{1}$ is normal and clearly $\cap \mathfrak{X}\left(A_{i}\right)=\mathfrak{X}\left(B_{1}\right)$. 
THEOREM 3.3. $\cap \mathfrak{A}\left(A_{i}\right)$ is p.w.m.

Proof. Let $A \supseteq B_{1}$ be a $\tau$-closed subgroup of $G$. Then $\mathfrak{A}(A) \subseteq \mathfrak{A}\left(B_{1}\right)$ is a RIC extension and by Theorem 1.1

$$
\mathfrak{S}\left(\mathfrak{X}\left(B_{1}\right) \cap \mathfrak{U}(A)^{\sharp}\right)=H(A) B_{1} \text {. }
$$

By Lemma 3.1 it is enough to show that $H(A) B_{1}=A$. However since $\mathfrak{U}\left(B_{1}\right) \cap \mathfrak{U}(A)^{\sharp} \supseteq \mathfrak{U}(A)$ this is equivalent to showing that

$$
\mathfrak{A}\left(B_{1}\right) \cap \mathfrak{A}(A)^{\sharp}=\mathfrak{A}(A) .
$$

Put $\mathscr{B}=\mathfrak{Y}\left(B_{1}\right) \cap \mathfrak{U}(A)^{\sharp}$; then $\mathfrak{Y}(A) \subseteq \mathscr{B}$ is a group extension and if $\mathscr{B} \neq$ $\mathfrak{A}(A), A / B \neq\{e\}$. There exists therefore a nontrivial homomorphism $\sigma$ of $\mathbf{Z}$ into $A / B$. Then by Proposition 3.2, $\operatorname{per}(\mathscr{B}, \sigma)$ is w.m., so there is $A_{i} \subseteq$ $\mathbb{B}(\operatorname{per}(\mathscr{B}, \sigma))$. Hence $\operatorname{per}(\mathscr{B}, \sigma) \vee \mathscr{B} \subseteq \mathfrak{A}\left(A_{i}\right)$ and is therefore w.m. But this contradicts the fact that $f_{\sigma}$ which is an almost periodic function belongs to the algebra $\mathscr{B} \vee \operatorname{per}(\mathscr{B}, \sigma)$. Thus $A / B=\{e\}, A=B$ and $\mathscr{A}(A)=\mathscr{B}$.

Corollary 3.4. $\mathscr{U}\left(B_{0}\right)=\cap \mathfrak{A}\left(A_{i}\right)$.

Proof. This follows immediately from Proposition 2.4, Theorem 3.3, and the maximality of $\mathfrak{A}\left(B_{0}\right)$.

Remark. All the material in $\$ 2$ as well as Propositions 3.1 and 3.2 are valid (with the obvious modifications) for an arbitrary group $T$ in place of the integers. For the proof of Theorem 3.3 we need the existence of a nontrivial homomorphism $\sigma: T \rightarrow A / B$. If $T$ is a free abelian group such a $\sigma$ always exists. Thus in Theorem 3.3 and Corollary $3.4 \mathrm{Z}$ can be replaced by any free abelian group.

LEMMA 3.5. Let $\mathbb{Q}$ be a P.O.D. algebra and $\mathscr{B} \supseteq \mathbb{Q}$ a group extension then T is regular.

PROof. By [7, Theorem 4.5], $|\mathfrak{Q}|$ is a regular flow and its automorphism group is $\left\{\tau^{n}: n \in \mathbf{Z}\right\}$ where $\tau$ is the generator of the action of $\mathbf{Z}$ on $|\mathbb{Q}|$. Let $\phi:$ $|\Re| \rightarrow|\mathbb{Q}|$ be the homomorphism from $|\Re|$ to $|\mathbb{Q}|$. Let $x, y \in|\Re|$ then there exists an $n$ such that $\phi(x) \tau^{n}$ is proximal to $\phi(y)$. Hence $\phi\left(x \tau^{n}\right)$ is proximal to $\phi(y)$ and for some $\kappa \in A / B(A=\mathbb{S}(\mathscr{Q}), B=\mathbb{S}(\mathscr{B})) x \kappa \tau^{n}$ is proximal to $y$. By [1, Theorem 4.3] $|\Re|$ is regular.

THEOREM 3.6. There does not exist a universal weakly mixing minimal flow. Equivalently, there exist maximal weakly mixing algebras $\mathfrak{Y}\left(A_{j}\right)$ and $\mathfrak{X}\left(A_{k}\right)$ such that $\mathfrak{A}\left(A_{j}\right) \alpha \neq \mathfrak{A}\left(A_{k}\right)$ for every $\alpha \in G$.

Proof. Let $\mathscr{Q}$ be a P.O.D. flow. Let $\mathscr{B} \supseteq \mathscr{Q}$ be a weakly mixing nontrivial group extension [8]. By Lemma $3.5 \mathscr{B}$ is regular i.e. $\mathscr{B} \alpha=\mathscr{B}$ for every $\alpha \in G$. Let $\mathscr{B} \subseteq \mathfrak{A}\left(A_{j}\right)$; since clearly $\mathscr{B}$ is not p.w.m. we have by Corollary 
3.4 $\mathscr{B} \nsubseteq \cap \mathfrak{X}\left(A_{i}\right)$, so that for some $k, \mathfrak{B} \nsubseteq \mathfrak{U}\left(A_{k}\right)$. It now follows that $\mathfrak{U}\left(A_{j}\right) \alpha \neq \mathfrak{A}\left(A_{k}\right)$ for all $\alpha \in G$.

REMARK. Let $X$ be a P.O.D. metric flow, let $Y$ and $Z$ be w.m. minimal group extensions of $X$ such that $Y \times Z$ contains a minimal set which is not w.m. [8]. Since both $Y$ and $Z$ are regular all minimal sets in $Y \times Z$ are isomorphic and thus none of them is w.m. This is a direct proof of Theorem 3.6.

4. None of the $A_{i}$ are normal. We let $\tau$ be the generator of the action of $\mathbf{Z}$. Recall that $\left\{A_{i}\right\}$ is the family of the minimal $\tau$-closed subgroups for which $\mathfrak{U}\left(A_{i}\right)$ is w.m.

LEMMA 4.1. Let $A \in\left\{A_{i}\right\}$ then $A$ is monothetic; i.e. there exists $\alpha \in A$ such that $A=\operatorname{gp}\{\alpha\}$ where $\operatorname{gp}\{\alpha\}$ is the $\tau$-closed subgroup of $G$ generated by $\alpha$.

Proof. Since $A E=G$ there exist $\alpha \in A$ and $\eta \in E$ with $\alpha \eta=u \tau$, whence $E \alpha=E \tau$. Let $B=\operatorname{gp}\{\alpha\}$. Then $B \subseteq A$ and $E B$ is a $\tau$-closed subgroup of $G$ containing $u \tau$. Hence $E B=G$ and so $B=A$.

Proposition 4.2. Let $A \in\left\{A_{i}\right\}$ then

$$
H(A)=A \cap E .
$$

Proof. Let $\alpha$ be a generator of $A$ with $E \alpha=E \tau$. Then $H(A) \alpha$ is a generator of the compact Hausdorff topological group $A / H(A)$. Hence there exists an epimorphism $\phi: G / E \rightarrow A / H(A)$ with $\phi(E \tau)=H(A) \alpha$. On the other hand the inclusion $A \hookrightarrow G$ induces an epimorphism $\psi: A / H(A) \rightarrow$ $G / E$ with $\psi(H(A) \alpha)=E \tau$. Thus $\phi=\psi^{-1}$ and so $\psi$ is an isomorphism. Hence ker $\psi=(A \cap E) / H(A)=\{H(A)\}$ and so $H(A)=A \cap E$.

RemarK. If $A$ is a $\tau$-closed subgroup with $H(A)=A \cap E$ then $A \hookrightarrow G$ induces an isomorphism $\psi$ of $A / H(A)$ into $G / E$. Hence $A / H(A)$ is abelian. Since $\mathbb{S}(\mathfrak{U}(A) \vee \mathscr{E})=A \cap E$ we have $\mathfrak{U}(A)^{\sharp}=\mathfrak{A}(A) \vee \mathcal{E}$. If moreover $A E$ $=G$ (i.e. $\mathfrak{X}(A)$ is w.m.) then $\left|\mathfrak{A}(A)^{\sharp}\right|=|\mathfrak{A}(A)| \times|\mathscr{E}|$ and $\psi$ is an isomorphism of $A / H(A)$ onto $G / E$.

LEMMA 4.3. Let $\mathbb{Q}$ be a w.m. algebra such that $|\mathfrak{Q}|$ is metric. Let $A=\mathbb{B}(\mathbb{Q})$, then $H(A) \neq A \cap E$.

Proof. By Peleg's result [8], there exists a nontrivial group extension $\mathscr{B} \supseteq \mathbb{Q}$ such that $\mathscr{B}$ is weakly mixing. Thus $B E=G$ and $H(A) \subseteq B$, where $B=\mathfrak{B S}(\mathscr{B})$. It follows that $B(A \cap E)=A$. If $H(A)=A \cap E$ then $B \cdot H(A)$ $=A$ and $B=A ;$ a contradiction.

LeMma 4.4. Let $A$ and $F$ be $\tau$-closed subgroups such that $A H(F)=F$ and $H(A)=A \cap E$. Then $H(F)=F \cap E$.

Proof. Let $\gamma \in F \cap E$. Then $\gamma=\alpha \beta$ with $\alpha \in A$ and $\beta \in H(F)$. Since 
$H(F) \subseteq F \cap E, \alpha=\gamma \beta^{-1} \in(F \cap E) H(F)=F \cap E$ and so $\alpha \in A \cap F$ $\cap E=A \cap E=H(A) \subseteq H(F)$. Thus $\gamma=\alpha \beta \in H(F) \cdot H(F)=H(F)$.

Corollary 4.5. Let $A$ and $B$ be $\tau$-closed subgroups such that $H(A)=A \cap$ $E, H(B)=B$ and $F=A B$ is a group. Then $H(F)=F \cap E$.

Proof. $A H(F)=A H(A B)=A H(B)=A B=F[5,3.12]$. (Note: It is easy to produce $B$ with $H(B)=B$; start with an arbitrary $\tau$-closed subgroup $L$ and set $B=L_{\infty}$ (see [5]). If moreover $L \triangleleft G$, then $L_{\infty} \triangleleft G$ and $F=A L_{\infty}$ is a group.) Recall that $\mathfrak{A}\left(B_{0}\right)$ is the maximum p.w.m. algebra.

Corollary 4.6. $H\left(B_{0}\right) \neq B_{0} \cap E$.

PRoof. Let $A$ be the group of the Furstenberg-Keynes-Shapiro prime flow [7]. Now $B_{0} \subseteq A$ and of course $B_{0} H(A)=A$. If $H\left(B_{0}\right)=B_{0} \cap E$ then $H(A)=A \cap E$. This is impossible by Lemma 4.3.

Question. For a $\tau$-closed subgroup $A$ of $G$, when does $H(A)=A \cap E$ ?

Let $\mathscr{B}$ be an algebra, $K$ a compact Hausdorff topological group. Let $\sigma \in Z(\mathscr{B}, K)$ and let $\mathcal{L}=\operatorname{ext}(\mathscr{B}, \sigma)$. The flow $|\mathcal{L}|$ is isomorphic to the orbit closure of $\left(e, x_{0}\right) \in K \times{ }_{\sigma}|\mathscr{B}|$. The subgroup $K_{0}=\{k \in K$ : $\left.\left(k, x_{0}\right) \in \overline{\left(e, x_{0}\right) \mathbf{Z}}\right\}$ is a closed subgroup of $K$ and $f_{\sigma}(B)=K_{0}$ where $B=$ (S)(B) . If $L=\operatorname{ker}\left(f_{\sigma} \mid B\right)$ then $\mathbb{S}(\mathcal{L})=L$ and $K_{0} \cong B / L$.

Lemma 4.7. Let $\mathscr{B}, K, \sigma, \mathcal{L}$ and $K_{0}$ be as above. Let $A \subseteq B$ be a $\tau$-closed group such that $H(A)=A \cap E$ then there exists $\rho \in \operatorname{Hom}\left(\mathbf{Z}, K_{0}\right)$ with $A \subseteq$ SS(per( $(\mathcal{L}, \rho))$.

Proof. $f_{\sigma}$ restricted to $A$ is $\tau$-continuous. Hence $H(A)=A \cap E \subseteq \operatorname{ker} f_{\sigma}$ and so $f_{\sigma}$ induces a homomorphism $\hat{f}_{\sigma}: A / A \cap E \rightarrow K_{0}$. The inclusion $A \hookrightarrow G$ induces an isomorphism $\phi: A / A \cap E \rightarrow G / E$. Set

$$
\rho(n)=\hat{f}_{\sigma}\left(\phi^{-1}\left(E \tau^{n}\right)\right) \quad(n \in \mathbf{Z}) .
$$

Then $\rho \in \operatorname{Hom}\left(\mathbf{Z}, K_{0}\right)$ and $f_{\rho}(\gamma)=f_{\sigma}(\alpha)(\gamma \in G)$, where $\alpha \in A$ satisfies $E \gamma=E \alpha$. Hence for $\alpha \in A$ we have $f_{\rho}(\alpha)=f_{\sigma}(\alpha)$. Now

$$
\mathbb{S}(\operatorname{per}(\mathcal{L}, \rho))=\left\{\beta \in B: f_{\rho}(\beta)=L \beta\right\} \text {, }
$$

but for $\alpha \in A, f_{\rho}(\alpha)=f_{\sigma}(\alpha)=L \alpha$ and hence $A \subseteq \mathbb{S}(\operatorname{per}(\mathcal{L}, \rho))$.

THEOREM 4.8. Let $A$ be a $\tau$-closed normal subgroup of $G$ with $A E=G$ and $H(A)=A \cap E$. Then $A=G$.

Proof. We first show that $A \supseteq B_{0}$. Let $P$ be a $\tau$-closed subgroup with $A \subseteq P$, by Lemma 3.1, we have to show that $A H(P)=P$. Suppose $A H(P)$ $\neq P$; let $F$ be a $\tau$-closed subgroup of $P$ such that $A H(P) \subset F$ and $K=$ $F / A H(P)$ is abelian. Let $\mathscr{B} \subseteq \mathfrak{A}(u)$ be the algebra which satisfies $\mathbb{S}(\mathscr{B})=B$ $=A H(P)$ and such that $\mathfrak{A}(F)=\mathscr{F} \subseteq \mathscr{B}$ is a group extension. Since $|\mathfrak{A}(F)|$ is 
0-dimensional there exists $\eta \in Z(\mathscr{F}, F / B)$ such that $\mathscr{B}=\operatorname{ext}(\mathscr{F}, \eta)$ and $f_{\eta}(\alpha)=B \alpha(\alpha \in F)[4 ; 4.15,4.5]$.

Let $\sigma \in Z(\Re, K)$ be defined by

$$
\sigma((k, x), \tau)=k \quad(x \in|\mathscr{|}|, k \in K),
$$

and let $\mathcal{L}=\operatorname{ext}(\mathscr{B}, \sigma)$. Thus $|\mathcal{L}|$ is isomorphic to the orbit closure of $\left(e, e, x_{0}\right)$ $\in K \times_{0}\left(K \times_{\eta}|\mathcal{F}|\right)$, where $x_{0}$ is the base point of $|\mathcal{F}|$ and the flow on $K \times_{0}\left(K \times_{\eta}|\mathscr{F}|\right)$ is defined by

$$
\left(k^{\prime}, k, x\right) \tau=\left(k^{\prime} k, k \eta(x, \tau), x \tau\right) \quad\left(k^{\prime}, k \in K ; x \in|\mathscr{F}|\right)
$$

We let

$$
K_{0}=\left\{k \in K:\left(k, e, x_{0}\right) \in \overline{\left(e, e, x_{0}\right) \mathbf{Z}}\right\} \text {. }
$$

Now apply Lemma 4.7 to obtain $\rho \in \operatorname{Hom}\left(\mathbf{Z}, K_{0}\right)$ with $A \subseteq \mathbb{S}(\operatorname{per}(\mathcal{L}, \rho))$. If $\rho(\tau)=k_{0} \in K_{0}$ then the flow on $|\operatorname{per}(\mathcal{L}, \rho)|$ is given by

$$
\left(k^{\prime}, k, x\right) \tau=\left(k_{0}^{-1} k^{\prime} k, k \eta(x, \tau), x \tau\right) \text {. }
$$

Let $\operatorname{per}(\mathcal{L}, \rho)=\mathscr{D}$; then $z_{0}=\left(e, e, x_{0}\right)$ is the base point of $|\mathscr{D}|$. Choose some point of the form $z_{1}=\left(k_{2}, k_{1}, x_{0}\right) \in|\mathcal{D}|$ with $k_{1} \neq e$. Then there exists $\alpha \in F \backslash B$ such that $\mathscr{D} \alpha$ is the algebra corresponding to the pointed flow $\left(|\mathscr{D}|, z_{1}\right)$. Let $\mathscr{R}=\mathscr{D} \vee \mathscr{D} \alpha$ then $|\mathscr{R}|$ is identified with $\overline{\left(z_{0}, z_{1}\right) \mathbf{Z}}$. Define $\phi$ : $|\Re| \rightarrow K$ by

$$
\phi\left(z, z^{\prime}\right)=\phi\left((k, l, x)\left(k^{\prime}, l k_{1}, x\right)\right)=k^{\prime} k^{-1}
$$

Then

$$
\begin{aligned}
\phi\left(\left(z, z^{\prime}\right) \tau\right) & =\phi\left(\left(k_{0}^{-1} k l, l \eta(x, \tau), x \tau\right)\left(k_{0}^{-1} k^{\prime} l k_{1}, l k_{1} \eta(x, \tau), x \tau\right)\right) \\
& =k_{0}^{-1} k^{\prime} l k_{1}\left(k_{0}^{-1} k l\right)^{-1}=k^{\prime} k^{-1} k_{1} \\
& =\phi\left(z, z^{\prime}\right) k_{1} .
\end{aligned}
$$

If $\chi$ is a character on $K$ such that $\chi\left(k_{1}\right) \neq 1$ then $\chi \circ \phi$ is an eigenfunction on $|\Re|$, and $|\Re|$ is not w.m. However,

$$
\mathbb{S}(\Re)=\mathbb{S}(\mathscr{D}) \cap \mathbb{S}(\mathscr{D} \alpha) \supseteq A \cap \alpha^{-1} A \alpha=A
$$

so that $\Re \subseteq \mathfrak{A}(A)$, a contradiction.

Thus we have $A \supseteq B_{0}$. Let $\mathscr{P} \subseteq \mathfrak{A}(A)$ with $|\mathscr{P}|$ metric and nontrivial. We have $A H(P)=P$ and $H(A)=A \cap E$ hence, by Lemma 4.4 we have also $H(P)=P \cap E$. This contradicts Corollary 4.6 and the proof is completed.

\section{THEOREM 4.9. None of the $A_{i}$ are normal.}

Proof. Follows immediately from Theorem 4.8.

REMARK. Starting with any metric w.m. algebra $\mathcal{F}$ and an abelian compact metric topological group $K$, there exists by [8] an $\eta \in Z(\mathcal{F}, K)$ such that 
$\mathscr{B}=\operatorname{ext}(\mathscr{F}, \eta)$ is weakly mixing and $|\mathscr{B}|=K \times_{\eta}|\mathscr{F}|$. Define $\sigma \in Z(\mathscr{B}, K)$ as in the proof of Theorem 4.8 and let $\mathcal{L}=\operatorname{ext}(\mathscr{B}, \sigma)$. If $A_{i} \subseteq B=\mathscr{S}(\mathscr{B})$ then as in 4.8 we can find $\rho \in \operatorname{Hom}\left(Z, K_{0}\right)$ for which $A_{i} \subseteq \&(\operatorname{per}(\mathcal{L}, \rho))$, and $\alpha \in F \backslash B$ such that $\mathscr{D} \vee \mathscr{D} \alpha$ is not w.m. where $\mathscr{D}=\operatorname{per}(\mathscr{L}, \rho)$. Thus $|\mathscr{D}|=$ $X$ is a metric w.m. minimal flow such that $X \times X$ contains a minimal subset which is not w.m.

\section{REFERENCES}

1. J. Auslander, Endomorphisms of minimal sets, Duke Math. J. 30 (1963), 605-614.

2. R. Ellis, Lectures in topological dynamics, Benjamin, New York, 1969.

3. —_ The construction of minimal discrete flows, Amer. J. Math. 87 (1965), 564-574.

4. Cocycles in topological dynamics (preprint).

5. R. Ellis, S. Glasner and L. Shapiro, Proximal-isometric flows, Advances in Math. 97 (1975), 148-171.

6. S. Glasner, Proximal flows, Lecture Notes in Math., vol. 517, Springer-Verlag, Berlin and New York, 1976.

7. H. Furstenberg, H. Keynes and L. Shapiro, Prime flows in topological dynamics, Israel J. Math. 14 (1973), 26-38.

8. R. Peleg, Some extensions of weakly mixing flows, Israel J. Math. 9 (1971), 330-336.

9. K. E. Petersen, Extensions of minimal transformation groups, Math. Systems Theory 5 (1971), 365-375.

School of Mathematics, Universtry of Minnesota, Minneapolis, Minnesota 55455

Department of Mathematical Sciences, Tel-Aviv University, Ramat-Aviv, TrL-Aviv, ISRARL 